\title{
Afecciones podales frecuentes en pacientes que asisten al consultorio podológico de la Facultad de Bioquímica y Ciencias Biológicas de la Universidad nacional de Litoral
}

Medrano, $\mathrm{P}$. 


\section{AFECCIONES PODALES FRECUENTES EN PACIENTES QUE ASISTEN AL CONSULTORIO PODOLÓGICO DE LA FACULTAD DE BIOQUÍMICA Y CIENCIAS BIOLÓGICAS DE LA UNIVERSIDAD NACIONAL DE LITORAL}

Medrano, Patricia

patri_medrano@hotmail.com

Facultad de Bioquímica y Ciencias Biológicas. Universidad Nacional del Litoral.

\section{RESUMEN}

El objetivo de este trabajo fue identificar las afecciones que se atienden en forma frecuente en el Consultorio de Podología de la Facultad de Bioquímica y Ciencias Biológicas de la Universidad Nacional del Litoral (FBCB-UNL) e investigar el perfil de los pacientes que asistieron a consulta desde mayo de 2015 a diciembre de 2018. Se planteó un trabajo de investigación cuantitativo, de carácter descriptivo transversal, para analizar los datos consignados en 430 Historias Clínicas Podológicas (HCP) correspondientes a todos los pacientes que concurrieron en este período de tiempo. Se construyó una base de datos en planilla de cálculo electrónica con la información obtenida. Los diagnósticos encontrados fueron: Hiperqueratosis (HQ), Onicomicosis (OM), Helomas (Hma), Onicocriptosis (OC), Onicofosis (OF), micosis interdigital, grietas talares y verrugas. Fue posible confeccionar el perfil del paciente tipo atendido, donde el sexo femenino es el que asiste con mayor frecuencia a consulta, en una franja etaria mayor de 36 años; está relacionado al ámbito universitario, realiza actividad física, usa calzado adecuado respecto a tacón bajo y cerrado; se acerca a la consulta con la inquietud de un corte de uñas, dolor en dedos, durezas, onicomicosis, no diabético, no alérgico, no hipertenso; predominio de pie normal y fórmula digital egipcia.

Palabras claves: consultorio Podológico, historia clínica, afecciones podológicas 


\section{SUMMARY}

\section{Frequent podal condicion in patient who attend in the podological office to Facultad de Bio- química y Ciencias Biológicas de la Universidad Nacional del Litoral}

The objective of this work was to identify the conditions that are frequently treated in the Podiatry Office of the Faculty of Biochemistry and Biological Sciences of the National University of the Litoral of the city of Santa Fe (FBCB) and to investigate the profile of the patients who attended the consultation from May 2015 to December 2018. A quantitative, descriptive, cross-sectional research work was proposed to analyze the data recorded in 430 Podiatric Medical Records (PCH) corresponding to all the patients who attended in this period of time. An electronic spreadsheet database was built with the information obtained. The diagnoses found were: Hyperkeratosis (HQ), Onychomycosis (OM), Helomas (Hma), Onychocryptosis (OC), Onychophosis (OF), interdigital mycosis, talar fissures and warts. It was possible to draw up the profile of the typical patient attended, where the female sex is the one who attends the consultation more frequently, in an age group older than 36 years, is related to the university environment, performs physical activity; wear appropriate footwear with respect to a low and closed heel; approaches the consultation with the concern of a nail cut, pain in fingers, hardness, onychomycosis, not diabetic, not allergic, not hypertensive; prevalence of normal foot and Egyptian digital formula.

Keywords: podriatic clinic, medical history, podriatic condition

\section{INTRODUCCIÓN}

La propuesta de este trabajo fue analizar la información contenida en las HCP del Consultorio de Podología de la FBCB para confeccionar una base de datos y obtener estadísticas de atención en el consultorio. Con este trabajo de investigación se logró un relevamiento de patologías frecuentes y perfil de personas atendidas, estableciendo antecedentes para futuros estudios de tipo estadístico o como base para realizar campañas de promoción y prevención sobre la salud del pie.

Entre las afecciones podológicas más conocidas podemos nombrar:

- Hiperqueratosis (HQ): es una hipertrofia o hiperplasia de la capa córnea por un aumento de queratina que cursa con un engrosamiento uniforme de la piel en una superficie amplia. Es el resultado de una alteración biomecánica que implica una zona que recibe más presión de la adecuada. Frecuentemente esta lesión se localiza en la zona plantar del antepié, bajo las cabezas metatarsales (2).

- Helomas (Hma): también llamados callos, son hiperqueratosis con un núcleo que se corresponde con un área de hiperpresión intermitente de la piel sobre un punto óseo. El mecanismo de producción se 
debe a la presión o a la fricción a la que se somete una determinada zona del pie donde se unen, por un lado, un relieve óseo o una exóstosis y, la acción externa del calzado. Se ubican en el dorso de los dedos, los espacios interdigitales y superficie plantar del antepié, en el punto donde existe máxima presión o cizallamiento de las cabezas metatarsales (2).

- Onicodistrofias: se refiere a toda alteración en la superficie ungueal, excluyendo el cambio de color. Son alteraciones frecuentes, y algunas de sus causas más comunes son los traumatismos de diferentes tipos (3).

- Hallux abductus valgus: es uno de los motivos de consulta más frecuente en mujeres dentro de las patologías que afectan el pie. Se caracteriza por deformidad en varo de primer metatarsiano y valgo del primer dedo, dolor en la prominencia ósea medial y debajo de cabeza del metatarsiano (4).

\section{Deformidad de los dedos}

- Dedo en garra: es aquel que presenta una hiperextensión de la articulación metatarsofalángica con flexión de la articulación interfalángica proximal y posición en flexión, de extensión o neutro de la interfalángica distal.

- Dedo en martillo: es aquella deformidad en la cual la articulación metatarsofalángica puede estar generalmente en hiperextensión o no, con flexión de la articulación interfalángica proximal y la articulación interfalángica distal encontrarse en posición de flexión, extensión o neutro (5).

Tabla 1: Afecciones dermatológicas más frecuentes y su correspondiente tratamiento aplicado por el profesional podólogo.

\begin{tabular}{|c|c|}
\hline Afecciones dermatológicas del pie & Tratamiento \\
\hline Helomas o callo & $\begin{array}{c}\text { Helotomía (Hmia): acción de enuclear y debridar núcleo de im- } \\
\text { plantación profundo en zona de hiperqueratósis. }\end{array}$ \\
\hline Hiperqueratosis o durezas & $\begin{array}{c}\text { Hiperquerectomía (HPQ): debridar en finas capas tejido córneo } \\
\text { de la piel que se encuentra engrosada debido a la compacta- } \\
\text { ción de células inertes color amarillento y consistencia dura. }\end{array}$ \\
\hline $\begin{array}{c}\text { Onicocriptosis o uña incarnada } \\
\text { pliegue ungular que genera malestar y puede producir infec- } \\
\text { ción. }\end{array}$ \\
\hline $\begin{array}{c}\text { Onicomicosis (OM) o infección por hongo } \\
\text { en las uñas }\end{array}$ & $\begin{array}{c}\text { Espiculotomía: acción de quitar el borde de uña incarnada en el } \\
\text { Interdisciplinar con Dermatólogo. }\end{array}$ \\
\hline $\begin{array}{c}\text { Onicofosis o formación de hiperqueratosis } \\
\text { en los surcos ungueales }\end{array}$ & Interdisciplinar con Dermatólogo. \\
\hline Verruga plantar & Debridar tejido engrosado en el surco ungueal \\
\hline
\end{tabular}

Fuente: elaboración propia. 


\section{MATERIAL Y MÉTODOS}

Se cotejó la totalidad de los registros existentes en las HCP disponibles en el Consultorio de Podología de la $\mathrm{FBCB}$, con el fin de identificar las variables y determinar las categorías de éstas que permitieron, luego de su análisis, la confección de una base de datos.

Ante la cantidad de información encontrada en 2 formatos diferentes de HCP y la falta de datos en algunas de ellas respecto a las variables analizadas, se tomó la decisión de trabajar con aquellos datos comunes a ambos registros, los cuales fueron: sexo, edad, ocupación, actividad deportiva, calzado frecuente, morfología del pie, diagnóstico, tratamiento y asiduidad de consulta.

Se procesó la información en una base de datos confeccionada con Microsoft Excel ${ }^{\circledR}$. Esto permitió determinar cuáles fueron las afecciones podológicas que, con mayor frecuencia, se atendieron en el consultorio, también se estableció si el motivo de consulta coincidió o no con el diagnóstico del profesional actuante y el porcentaje de pacientes que utilizaron el servicio de podología por año.

\section{RESULTADOS Y DISCUSIÓN}

De las 430 HCP analizadas, se observó que 99 registraron sexo masculino y 331 sexo femenino. Como se puede observar, asistieron al consultorio más mujeres que varones, en una relación de aproximadamente 3,34 mujeres por cada hombre.

Este resultado se explica, si observamos el trabajo de otros autores cuyos estudios incluyeron muestras que resultaron en mayoría de mujeres sobre hombres, como es el caso de Manso et. al. (5), al estudiar la frecuencia de patologías podológicas relacionadas a queratopatías, o Spink et. al. (6), quienes investigaron sobre el uso del calzado con tacón desde jóvenes causando patologías como hallux valgus o deformidad en los dedos, los cuales concluyeron que las mujeres resultan más propensas a ciertas afecciones podológicas. También podemos tener en cuenta la investigación de Fillingim et. al. (7) quienes observaron que las mujeres obtienen respuestas experimentales al dolor clínicamente más relevantes que los hombres, lo que las impulsa a una consulta más frecuente.

Se encontró que la edad mínima de paciente atendido fue 11 años y la edad máxima 92 años. El rango de edad para el sexo femenino varió de un mínimo de 11 años a un máximo de 80 años, en tanto que para el sexo masculino fue de 13 a 92 años. La mayoría de las personas que acudieron a consulta se situó en el rango de 36 a 64 años, en semejanza con lo investigado por López López et al. (8), donde se obtuvo una muestra que oscilaba entre 41 a 60 años.

Se pudo observar que más de la mitad de las personas atendidas están relacionadas con el ámbito universitario (53\%), esto se explica con el hecho de que el Consultorio Podológico está enclavado en un punto central del desarrollo universitario de la ciudad, donde se encuentran 7 facultades de las 10 que posee la Universidad 
Nacional del Litoral en el ejido urbano, específicamente en el segundo piso de la Facultad de Bioquímica y Ciencias Biológicas. El 29\% fue ajeno al ámbito universitario y por último se encontró con $18 \%$ de HCP donde ese ítem no tiene registro.

El $50 \%$ de los pacientes manifestó que realizaba actividad deportiva, en tanto un $17 \%$ indicó que no. Existe un 33\% del que no se tiene este dato. Este relevamiento difiere de lo investigado por López López et. al. (8) quienes, sobre su grupo de estudio, encontraron $45 \%$ de conductas sedentarias como leer, escuchar música o mirar televisión; y además la IV Encuesta Nacional de Factores de Riesgo del Instituto Nacional de Estadística y Censos (9), la cual indicó en sus considerandos un aumento de inactividad física como factor de riesgo para enfermedades no transmisibles en Argentina.

De las $430 \mathrm{HCP}$ analizadas se observó que $72 \%$ manifiesta la utilización de taco bajo (de acuerdo a lo recomendable), $6 \%$ taco alto y $22 \%$ no registra este dato. Estos registros concuerdan con los resultados de Menz et. al. (10), cuya investigación concluye que el calzado con tacón cerrado, cierre ajustado y suela firme es óptimo para equilibrio y marcha. López López et. al. (8), en su grupo de estudio encontraron que la conducta preventiva más repetida e importante para el cuidado del pie consistió en acudir al podólogo, utilizar un calzado adecuado y mantener la higiene.

En las HCP se observó para ambos pies un $34 \%$ de tipo de pie normal, $26 \%$ pie cavo y $13 \%$ pie plano, guardando relación con estudios de Zurita Ortega et. al. (11) con la diferencia de que este autor realizó su investigación sobre una muestra de individuos jóvenes y deportistas, se encontró $1 \%$ de pie plano valgo y $26 \%$ no consignó este dato (Gráfico 1).

Gráfico 1: Porcentajes de los tipos de pies registrados en la muestra analizada

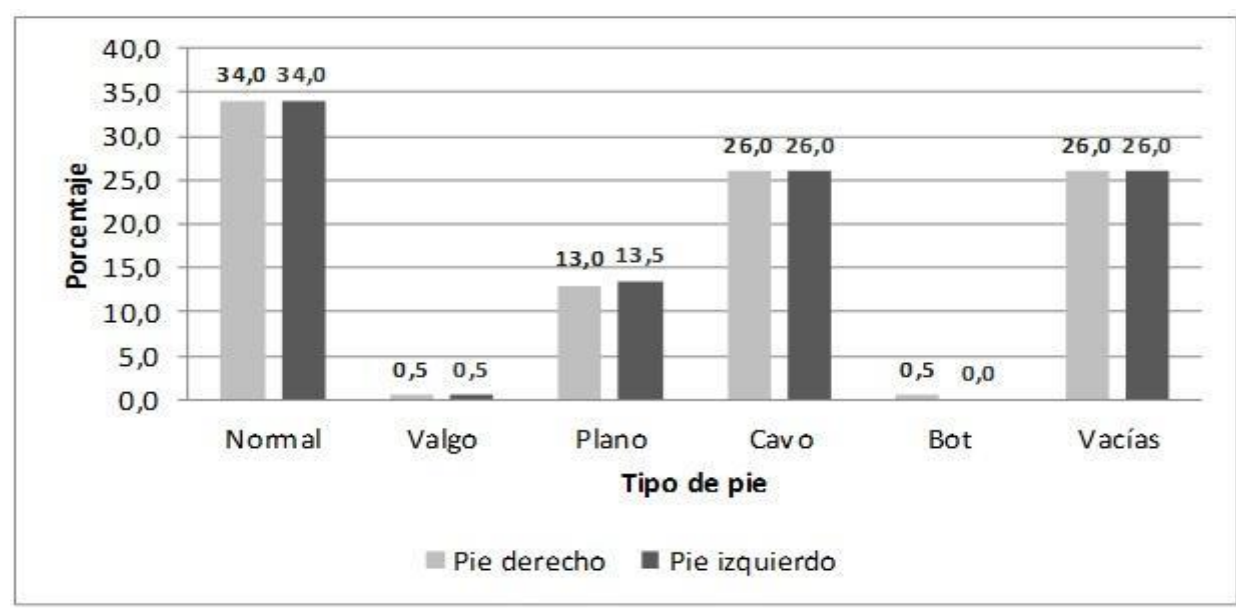

Fuente: elaboración propia.

En la muestra analizada predomina la fórmula digital egipcia con 56\% (primer dedo más largo que el segundo o antepié egipcio), 15\% griega (el primer dedo es más corto que el segundo o antepié griego) y $6 \%$ 
romana (primer y segundo dedos iguales o antepié romano); $23 \%$ no consignó este dato (Gráfico 2). Estos hallazgos coinciden con el estudio de Pérez Pico et. al. (12) donde señala el predominio de pie egipcio, pero en segundo lugar se encuentra la fórmula romana y en tercer lugar la griega.

Gráfico 2: Porcentajes de las fórmulas digitales registradas en la muestra analizada

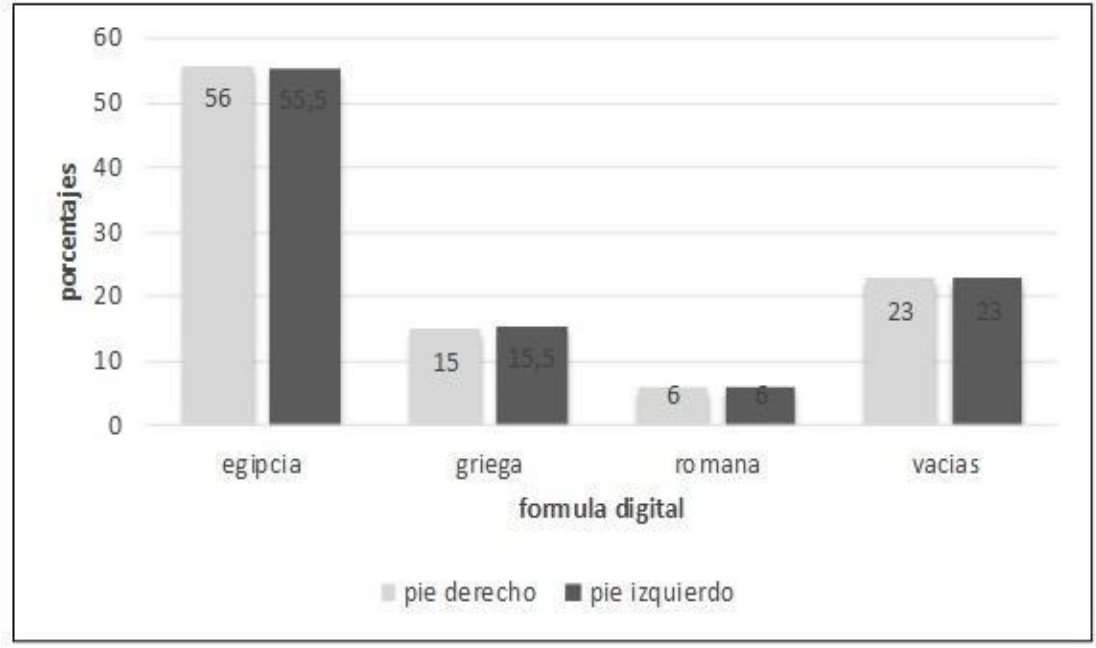

Fuente: elaboración propia.

En esta investigación, se observó que el profesional actuante determinó un diagnóstico prevalente de hiperqueratosis $41 \%$, en coincidencia con la patología de prevalencia encontrada en la investigación de Ochaita et. al. (13), luego le siguió onicomicosis 13\%, Helomas 11\%, Onicocriptosis $9 \%$, Onicofosis $6 \%$, Micosis Interdigital, grietas talares y verrugas $7 \%$; mientras que en el $13 \%$ no se encontró registro (Gráfico 3). Tamayo et. al. (14) encontraron que en las dos terceras partes de su muestra estudiada los pacientes acudían a consulta haciendo referencia a hiperqueratosis. Por el contrario, López Abad et. al. (15), en su estudio sobre una pequeña muestra de personas adultas de edad media, encontró que la OM tenía un alto grado de prevalencia a diferencia de lo encontrado en el presente estudio. 
Gráfico 3: Porcentajes de las afecciones podológicas encontradas en la muestra analizada

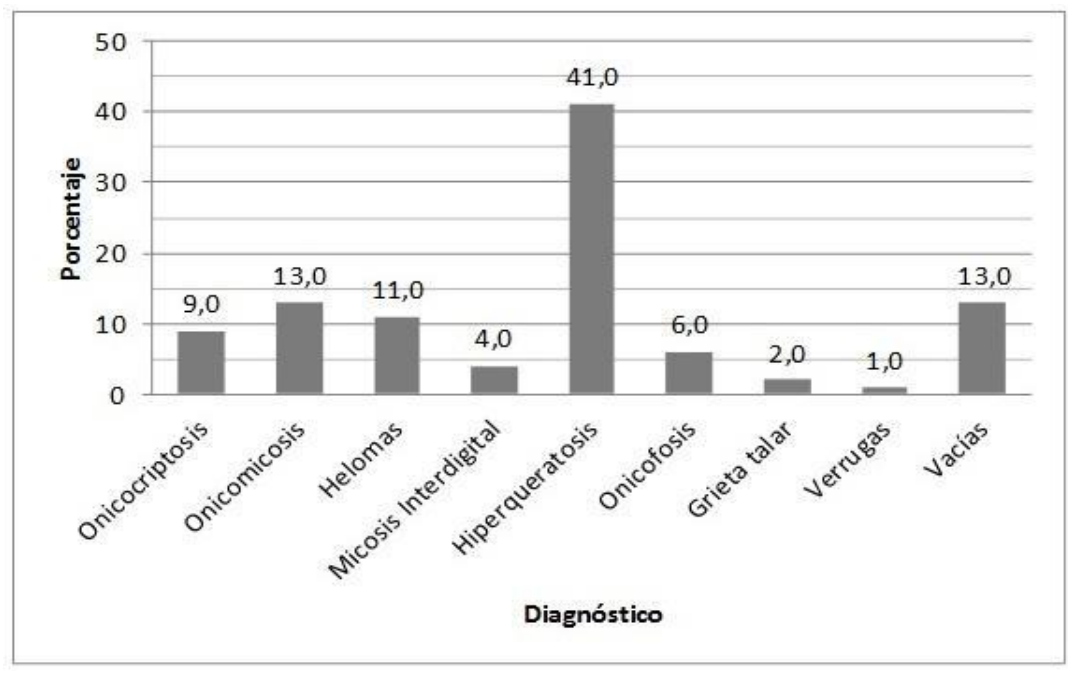

Fuente: elaboración propia.

En la práctica de una quiropodia (procedimiento podológico), se brinda tratamiento a toda aquella afección del pie requerida para mantener la salud podal, por lo que pueden proceder varios tratamientos en un mismo paciente y en una sola consulta. La onicotomía (OMIA), la cual se refiere al corte de uñas, se requirió en un 96\%, ésta es una práctica habitual que se realiza al paciente a menos que el profesional actuante no lo considere necesario. Se aplicó Hiperquerectomía (HPQ) en 51\%, siguiéndole Helotomía (HMIA) en 13\%, fresado por onicomicosis $10 \%$, espiculotomía de 3ำ en 8,5\%, eliminación de onicofosis (OF) en $5 \%$ y por último tratamientos específicos de puentes blandos (teflón) para reeducación del surco y equilibrar la estructura onicodactilar (Gráfico 4). Se infiere que la diferencia en porcentajes entre el tratamiento HQ - HPQ; Hma - Hmia; OM - fresado por onicomicósis, corresponde a la atención integral del pie, ya que aún cuando el profesional determina un diagnóstico y un tratamiento, se asiste toda aquella patología que necesita el pie para lograr salud.

Gráfico 4: Porcentajes de tratamientos aplicados a los pacientes de la muestra analizada

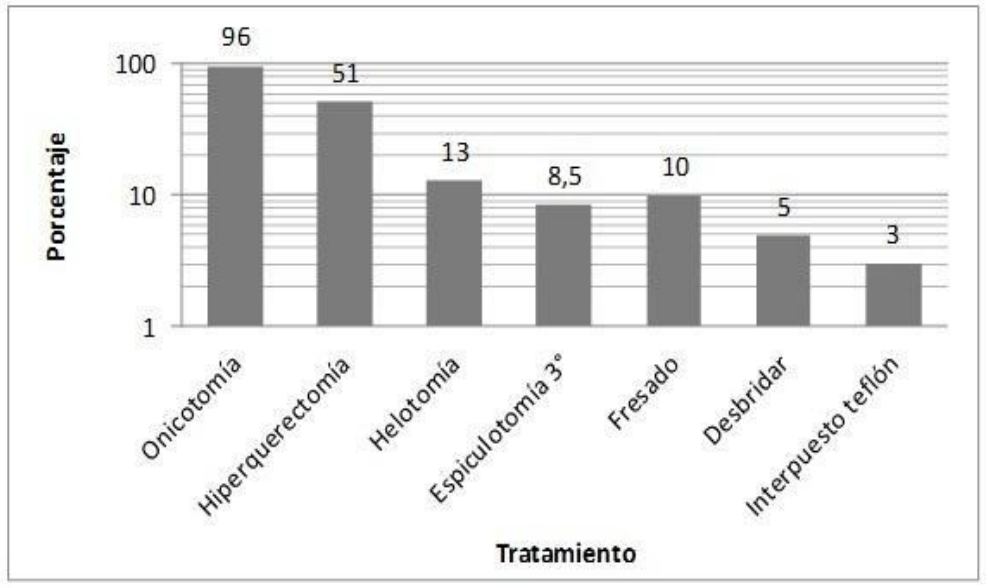

Fuente: elaboración propia. 
En la cantidad de nuevos pacientes que se registraron por año, durante el tiempo de investigación, se observó que el 31\% de las atenciones correspondieron al año 2015, cuando se inició con las atenciones en el Consultorio de Podología. En los años sucesivos se manifestó una pequeña merma, alcanzando una meseta alrededor del 23\% promedio, 26\% en 2016, 21\% en 2017 y, finalmente, 22\% en 2018 (Gráfico 5).

Gráfico 5: Porcentajes de pacientes atendidos por año en el período de tiempo analizado

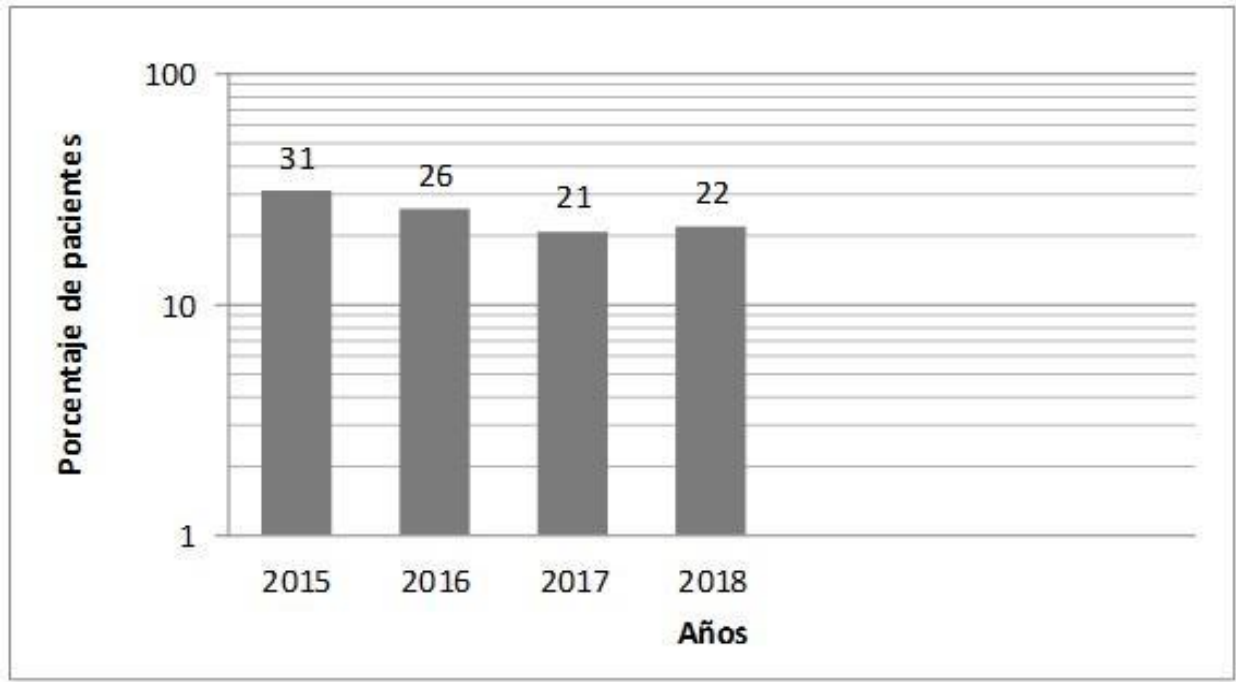

Fuente: elaboración propia.

\section{CONCLUSIÓN}

Del análisis de la información contenida en las HCP, se pudo conocer las afecciones podales frecuentes en el Consultorio de Podología de la FBCB, con diagnóstico prevalente de acuerdo con el siguiente orden: $41 \% \mathrm{Hi}$ perqueratosis, seguido de $13 \%$ Onicomicosis, $11 \%$ Helomas, $9 \%$ Onicocriptosis y $6 \%$ Onicofosis, luego micosis interdigital, grietas talares y verrugas.

Perfil del paciente según los datos analizados:

- El sexo femenino es el que asiste con mayor frecuencia a consulta.

- Franja etaria mayor de 36 años.

- En su mayoría, es un paciente relacionado al ámbito universitario (53\%).

- Paciente que realiza actividad física.

- Utiliza calzado adecuado; respecto a taco bajo y cerrado.

- Se acerca a la consulta con la inquietud de un corte de uñas, pero preocupado también por callosidades, dolor en dedos, durezas, onicomicosis.

- No diabético, no alérgico, no hipertenso.

- Prevalencia de pie normal, fórmula digital egipcia. 
Los problemas en los pies son considerados como un factor de riesgo para la movilidad y más aún en adultos mayores donde el avance de la edad aumenta los riesgos de caídas en razón del deterioro del equilibrio. En esta investigación se encontró que el grupo etario de adultos y tercera edad es el que acude a consulta con asiduidad, se considera conveniente promover un trabajo interdisciplinario entre las carreras de Licenciatura en: Nutrición, Enfermería, Educación Física y Podología, todas dependientes de la Facultad de Bioquímica y Ciencias Biológicas, con el fin de generar un espacio o programa para colaborar con este colectivo en su interés de salud.

Por otra parte, sabiendo que Podología es salud del pie, se cree necesario una campaña de promoción y prevención desde las áreas mencionadas, destinada a todos los estudiantes de Ciudad Universitaria y extensiva a la comunidad, que se infiere involucraría una franja etaria menor a la de mayor asistencia encontrada en esta investigación, para enseñar el correcto cuidado del pie y además, realizar evaluaciones podológicas. Esto último, podría ayudar a mejorar la salud podal a futuro, y que estos jóvenes de hoy se conviertan en adultos mayores con un hábito de cuidado del pie incorporado.

\section{BIBLIOGRAFÍA}

1-Prats Climent B., 2003. Alteración local de las partes blandas. Rev. Esp. Reumatol 30,9: 503 - 507.

2-Cardona H; Lazo G., 2015. Onicodistrofia traumática. Presentación de dos casos. Rev cent Dermatol Pascua. 1,24: 21-27.

3-Reyes Rodríguez C; Bordas Sanchez D. A.; Bustamante Rubio M. A.; Garcia F.; Mejia L. S., 2019. Etiología y fisiopatología del hallux valgus. Rev Colombiana de Ortopedia y Traumatología. 33,3: 2-12.

4-Pastrana García F.; Olivares Gutiérrez J.; Bárcena Jiménez L.; Fuentes Nucamendi M., 2008.Tratamiento de la deformidad en garra de los dedos menores del pie. Acta ortopédica mexicana. 22,3: 189 -194.

5-Manso P.; Borreiros F.; Soto M.; Fernández A.; Cotelo M. C.; Martin M., 2010. Patología de los pies y características sociodemográficos de la población usuaria de los centros sociales de mayores de Galicia. Rev. Podológica El Peu. 30,4:176 -183.

6-Spink M. J.; Menz H. B.; Lord S. R., 2009. Distribution and correlates of plantar hyperkeratotic lesions in older people. J Foot Ankle. 2,8:1-7.

7-Fillimgim R. B.; Edwards R. R.; Powell T., 1999. La relación del sexo y el dolor clínico con las respuestas experimentales al dolor. PAIN. 83,3: 419-425.

8-López López D.; García Mira.; Alonso Tajes F.; López López. L., 2010. Análisis del perfil y estilo de vida de las personas con patologías en los pies. Revista Internacional de Ciencias Podológicas 4,2: 49 - 58. 
9-Instituto Nacional de Estadística y Censos (INDEC)., 2019. IV Encuesta Nacional de Factores de Riesgo. Secretaría de Gobierno de Salud. Disponible en: https://www.indec.gob.ar/ftp/cuadros/publicaciones/enfr 2018 resultados preliminares.pdf

10-Menz H. B.; Auhl M.; Munteanu S. E., 2017. Effects of Indoor Footwear on Balance and Gait Patterns in Community-Dwelling Older Women. Gerontology. 63: 129-136.

11-Zurita Ortega F.; Martínez Martínez A.; Zurita Ortega A.; 2007. Influencia de la tipología del pie en la actividad físico-deportiva. Fisioterapia 29,2: 74 - 9.

12-Pérez Pico A. M.; Castaño Justo B.; Mayordomo Acevedo R., 2016. Relación entre la fórmula digital y las deformidades del antepié en una población joven. Eur. Jor. Pod. 2,1:1-11.

13-Ochaita P.; Llera F.; Marti V.; Megias A.; Vilanova M.; Castella J., 2004.Trastornos dermatológicos y podológicos en los pies de las personas mayores de 60 años. Piel. 19,4: 184-190.

14-Tamayo J.; González M.; Ramos R., 2007. Hiperqueratosis palmo-plantar. Frecuencia en el Hospital General de Ciudad Victoria, Tamps. Dermatología CMQ.5,2: 75 -79.

15-Lopez Abad S.; Mosquera Fernandez A.; Monteagudo Sánchez B., 2014. Prevalence of skin and nail pathology in a population sample of a geriatric centre from the province of a Coruna Enferm Dermatol. 8,23: $15-21$. 